\title{
Beyond the Galaxy Luminosity Function
}

\author{
Simon Driver ${ }^{\mathrm{A}}$ \\ A Mount Stromlo Observatory, Weston Creek ACT 2902, Australia. Email: spd@mso.anu.edu.au
}

Received 2004 April 3, accepted 2004 August 25

\begin{abstract}
With the advent of large-scale surveys (i.e. Legacy Surveys) it is now possible to start looking beyond the galaxy luminosity function (LF) to more detailed statistical representations of the galaxy population, i.e multivariate distributions. In this review I first summarise the current state-of-play of the $B$-band global and cluster LFs and then briefly present two promising bivariate distributions: the luminosity-surface brightness plane (LSP) and the colour-luminosity plane (CLP). In both planes galaxy bulges and galaxy disks form marginally overlapping but distinct distributions, indicating two key formation/evolutionary processes (presumably merger and accretion). Forward progress in this subject now requires the routine application of reliable bulge-disk decomposition codes to allow independent investigation of these two key components.
\end{abstract}

Keywords: galaxies: fundamental parameters — galaxies: luminosity function — galaxies: mass function — galaxies: statistics — surveys

\section{Introduction}

For almost 30 years the Schechter luminosity function (LF; Schechter 1976) has been the standard tool for quantifying the galaxy population ${ }^{1}$. The LF is loosely based on the Press-Schechter formalisation for the primordial halo distribution (Press \& Schechter 1974). Moreover the LF consistently provides a good formal fit to the observed luminosity distribution (LD; see for example Norberg et al. 2002). This consistency between the LD and LF appears to hold regardless of environment (De Propris et al. 2003; Driver \& De Propris 2003). The only departure from a pure Schechter function appears to be in the central cores of rich clusters, where the galaxy LD is often seen to show a marked upturn at the giant-dwarf boundary $\left(M_{B} \approx-16 \mathrm{mag}\right)$. Perhaps the most well known example is the central LD of the Coma cluster (e.g. Trentham 1998; Beijersbergen et al. 2002; Andreon \& Culliandre 2002 and references therein). The most plausible explanation is that the core contains an overdensity of giant and dwarf ellipticals bolstering both the bright- and faint-end of the core cluster LF. For example the more extensive Coma survey by Mobasher et al. (2004) recovers a flat and invariant $\mathrm{LD} / \mathrm{LF}(\alpha=-1)$ to $M_{B} \approx-14 \mathrm{mag}$. The phenomena of an upturn in the LD has also been seen in Virgo (Impey \& Bothun 1988; Trentham \& Hodgkin 2002), A963 (Driver et al. 1994), A868 (Driver et al. 2003), A2554 (Smith et al. 1997), and A2218 (Pracey et al. 2004) for example. However, apart from these 'active' core environments, the overall LDs from the field, to the local group, to the local

\footnotetext{
${ }^{1}$ The Schechter function $d(\phi)=\phi^{*}\left(L / L^{*}\right)^{\alpha} e^{\left(-L / L^{*}\right)} d\left(L / L^{*}\right)$ has three key parameters, being $L^{*}$ the characteristic luminosity where the exponential cutoff cuts in, $\phi^{*}$ the normalisation at this characteristic luminosity, and $\alpha$ the faint-end slope parameter. A value of $\alpha=-1$ implies equal numbers of galaxies in magnitude intervals, a more negative (or steep) value implies numerous dwarf systems.
}

sphere, and near and far rich clusters, all consistently follow a smooth LF within the luminosity ranges probed. Figure 1 shows an (incomplete) summary of $b, B, V$, or $g$ band field and cluster LFs colour-corrected to the Johnson $B$-filter.

The main point to take from Figure 1 is that the global and cluster LFs each show a broad but overlapping range of distributions. Clearly one cannot reasonably argue for any significant variation between the global and overall cluster environment on the basis of these data. Studies based within the same survey data, for example the TwoDegree Field Galaxy Redshift Survey (2dFGRS) study by Croton et al. (2004), generally find fairly subtle changes with environment. Hence it seems that the variations seen in Figure 1 indicates an unspecified systematic error in the various studies. The most lauded of these is the unsavoury topic of surface brightness selection effects (Disney 1976; Impey \& Bothun 1997). The concern is that the galaxy population at each luminosity interval occupies a range in surface brightness (or size). Surveys with shallow detection isophotes may miss both light from a galaxy's halo, as well as entire galaxies (see for example Sprayberry et al. 1997 and Dalcanton 1998). Cross \& Driver (2002) explored this possibility in detail and demonstrated that indeed surface brightness selection effects can play havoc with the recovered Schechter function parameters and reproduce exactly the kind of variation seen in both the global and cluster LFs of Figure 1.

More recently a number of papers have identified a clear luminosity-surface brightness (or size) ${ }^{2}$ relation for

\footnotetext{
${ }^{2}$ Luminosity, size, and surface brightness are related by $\mu_{\mathrm{e}}=M+2.5 \log _{10}\left[2 \pi R_{\mathrm{HLR}}^{2}\right]+36.57$ where $\mu_{\mathrm{e}}$ is the effective surface brightness, $M$ the absolute magnitude, and $R_{\mathrm{HLR}}$ the semi-major axis half-light radius in kpc, hence the luminosity-surface brightness relation can be readily transformed to a luminosity-size distribution and we use the acronym LSP to indicate either.
} 


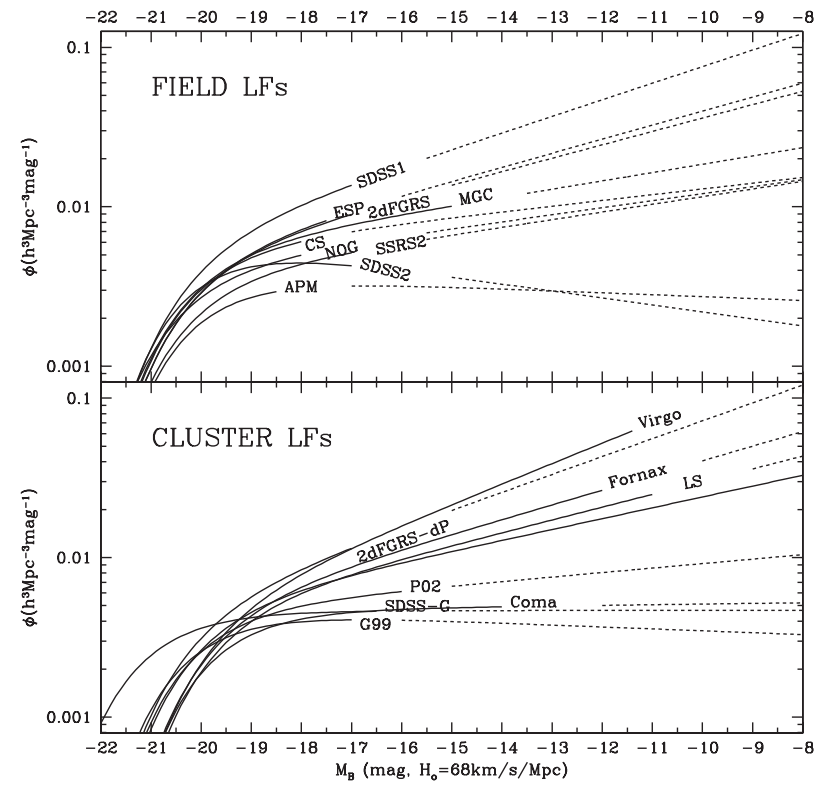

Figure 1 Various luminosity functions as measured for the global environment (top) and cluster environment (bottom). Data are taken from table 3 of Liske et al. (2003), table 1 of Driver \& De Propris (2003), tables $2 \& 3$ from Blanton et al. (2003), and table 2 from Driver et al. (2004). The solid lines show the regions over which the luminosity functions have been fitted and the dotted lines the extrapolations. The cluster luminosity functions have all been arbitrarily normalised to $\phi^{*}=0.0161$ galaxies per $h^{3} \mathrm{Mpc}^{-3}$.

field galaxies based on diverse datasets including the Hubble Deep Field (Driver 1999), the 2dFGRS (Cross et al. 2001), the Sloan Digital Sky Survey (SDSS; Blanton et al. 2001; Shen et al. 2003), and a very local inclination and dust corrected sample of late-type disks (de Jong \& Lacey 2002). These studies consistently show that low surface brightness is synonymous with low luminosity - with a few notable exceptions as typified by Malin 1 (Bothun et al. 1987) and the faint second disk surrounding NGC5084.

To fully resolve the potential impact of surface brightness selection effects one must consider the joint luminosity-surface brightness distribution. This has been advocated in the past, not so much to compensate for selection bias but to preserve the size (or surface brightness) information which may be of interest in its own right (see Chołoniewski 1985 and Sodré \& Lahav 1993 for instance). This latter point is illustrated in Figure 2, where I show an example LF for a nearby volume limited sample and images of the actual galaxies contributing to the LF. Clearly much information is lost when one replaces these images with three simple numbers. It is for these reasons - the need to accommodate selection bias and the desire to explore additional parameter space - coupled with the abundance of data that now moves us beyond the simple LD/LF to start exploring multivariate distributions. Here I introduce two such distributions, the luminositysurface brightness plane (for the reasons stated above) and the colour-luminosity plane, which is also of topical interest (e.g. Baldry et al. 2004; Hogg et al. 2004 and references therein).

\section{Multivariate Distributions}

To construct multivariate distributions requires an extensive wide area survey $\left(>10^{\circ}\right)$ to reasonable depth $\left(\mu_{\text {lim }}>25 B\right.$ mag $\left.\operatorname{arcsec}^{-2}\right)$, with reasonable resolution $\left(\mathrm{FWHM}=1^{\prime \prime}\right)$, wavelength coverage (e.g. some of $u b_{J} B V g r R i I_{c} z J H K$ etc. $^{3}$ ), and spectroscopic redshifts/ distances. The most notable catalogues for this purpose are the $2 \mathrm{dFGRS}\left(b_{j} R_{F}, 1800 \mathrm{deg}^{2}, 250000 z\right.$ 's; Colless et al. 2001), the SDSS (ugriz, $10000 \mathrm{deg}^{2}, 10^{6} z$ 's; Stoughton et al. 2002), the Millennium Galaxy Catalogue (MGC; $B+$ SDSS, $37 \mathrm{deg}^{2}, 10^{4} z$ 's; Liske et al. 2003), and the two Micron All Sky Survey (2MASS; $J H K$, all sky, SDSS $+2 \mathrm{dF}+6 \mathrm{dF}+\mathrm{MGC} z$ 's; Jarrett et al. 2003). The MGC, although the smallest in area, is also the deepest $\left(\mu_{\mathrm{lim}}=26.0 \mathrm{~B}\right.$ mag $\left.\operatorname{arcsec}^{-2}\right)$, highest resolution $\left(\mathrm{FWHM}=1.25^{\prime \prime}\right)$, and most complete survey (see Liske et al. 2003; Cross et al. 2004; Driver et al. 2004). It also overlaps with the other three surveys and hence provides a 'best of all worlds' hybrid dataset - for example $50 \%$ of the $\sim 10000$ MGC redshifts derive from the 2dFGRS or SDSS, extensive optical colour coverage from SDSS, and partial near-IR coverage from 2MASS. The $\mathrm{MGC}^{4}$ contains 10061 resolved galaxies with $12.5<B_{\mathrm{MGC}}<20$ mag with $95 \%$ complete redshift coverage. All galaxies have been analysed with a variety of software packages including SEXTRACTOR (Bertin \& Arnouts 1996), GIM2D (Simard et al. 2002), and eyeball-classified to $B_{\mathrm{MGC}}<19 \mathrm{mag}$.

\subsection{The Luminosity-Surface Brightness Plane}

The luminosity-surface brightness plane (LSP) is of particular interest because it enables one to compensate for both luminosity (Malmquist bias) and surface brightness selection effects (also known as 'Disney bias'). In Driver et al. (2004) the LSP is derived for the MGC, which provides the most robust current estimate. The MGC LSP analysis used the joint luminosity-surface brightness Step-Wise Maximum Likelihood method of Sodré \& Lahav (1993) and incorporates into this tracking of five selection boundaries relevant to each individual galaxy (i.e. maximum and minimum observable size and flux, and minimum observable central surface brightness for detection; see Driver 1999). An additional feature is the derivation of individual $K$-corrections using the combined MGC and SDSS-DR1 colours (u Bgriz). Figure 3 shows the data as a series of Gaussian fits across the LSP at progressive intervals of absolute magnitude. The thicker lines shows the surface brightness distribution for the most luminous galaxies and the fainter lines for the dwarf regime. Two facts leap out. Firstly, the distributions are bounded (the Gaussian fits have good $\chi^{2}$ values) with broadening towards lower luminosity.

\footnotetext{
${ }^{3}$ We specifically limit ourselves to the optical/IR regime but note the existence of all sky Hi, X-ray, and far-IR surveys.

${ }^{4}$ The MGC imaging and basic catalogues are available from www.eso.org/ jliske/mgc/ (additional catalogues including redshift, morphological, and structural parameters are available on request from the author).
} 


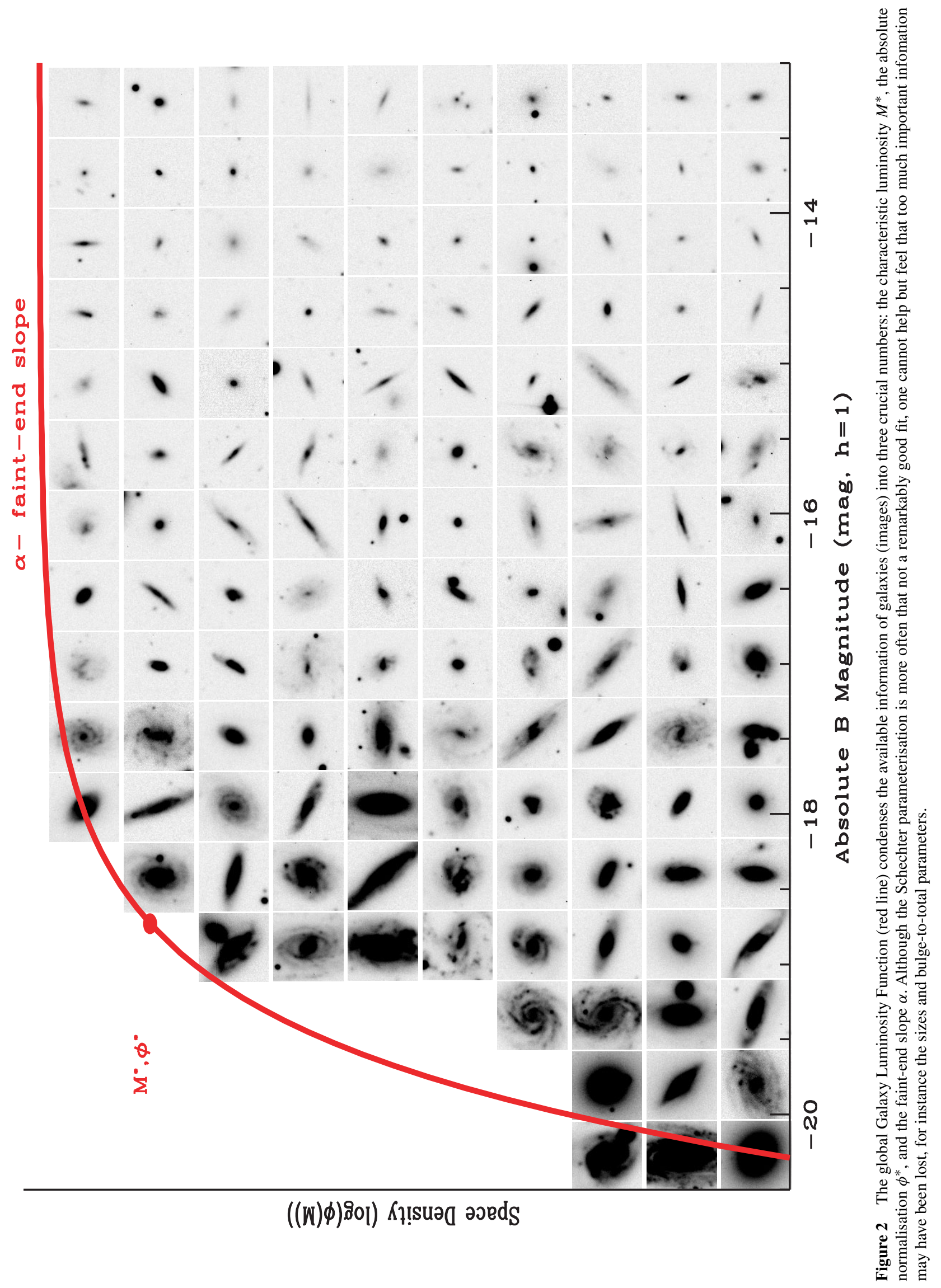




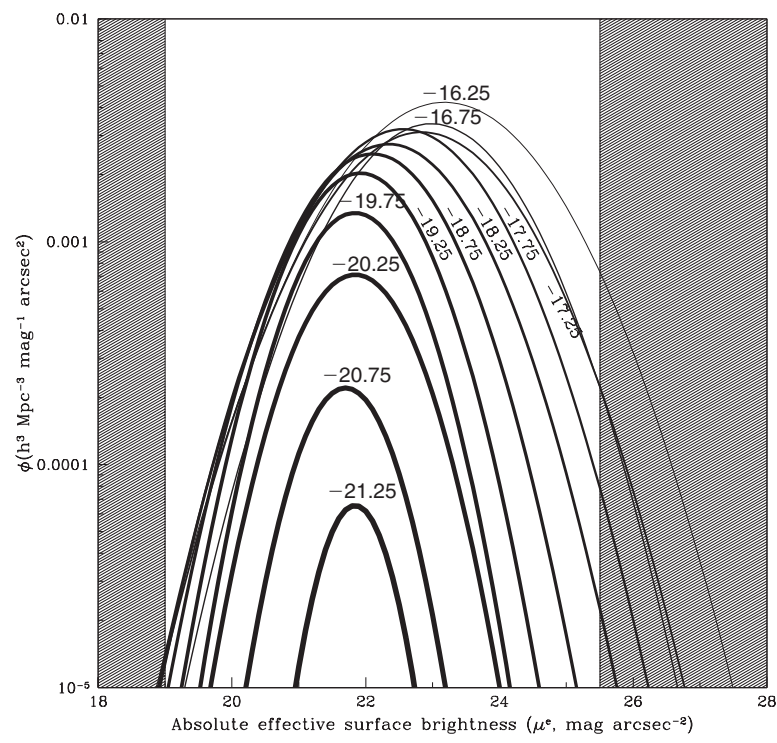

Figure 3 The surface brightness distribution of galaxies at various luminosity intervals (as indicated). The curves show the Gaussian fits to the recovered joint luminosity-surface brightness distribution of Driver et al. (2004). The shaded region denotes the limits at which strong selection effects are likely to impact upon the observed distributions. Generally the distribution is narrow and constant for the brightest galaxies (also known as Freeman's Law) and then broadens towards lower surface brightness for lower luminosity systems.

Secondly, the peak of the distribution moves towards lower surface brightness for lower luminosity systems. In other words, low luminosity systems apparently show greater diversity in surface brightness than giant systems. However this can also be interpreted in terms of the Kormendy relation for spheriods (Kormendy 1977) and Freeman's Law for disks (Freeman 1970). These two classic studies unveiled distinct relations for the structural properties of spheroid and disk components. The Kormendy study found that the more luminous the spheroid the lower its central surface brightness. Conversely Freeman's study found that all disks, regardless of luminosity, have a constant central surface brightness of $\mu_{B \mathrm{MGC}}^{\circ}=21.65 \pm 0.3 \mathrm{mag} \mathrm{arcsec}^{-2}$. The MGC results shown on Figure 3 are for the combined bulge+disk systems. Around $L^{*}$ the effective surface brightness for spheriods and disks is fairly close - a long-time nagging coincidence. However moving towards lower luminosity the trends for spheriods and disks diverge, leading to the broadening of the global surface brightness distribution. To investigate further hence requires separating out these two structural components through two-dimensional bulge-disk decomposition. Here we use GIM2D (Simard et al. 2002) and Figure 4 shows the data of Figure 3 subdivided by structural component. The dotted line shows the original Freeman distribution which remains relevant today, albeit with a far broader dispersion than originally reported (see Freeman 1970). It would seem that galaxies consist of two principle components (presumably formed by means of two mechanisms, merging and accretion/collapse) and to unravel these two phases in detail
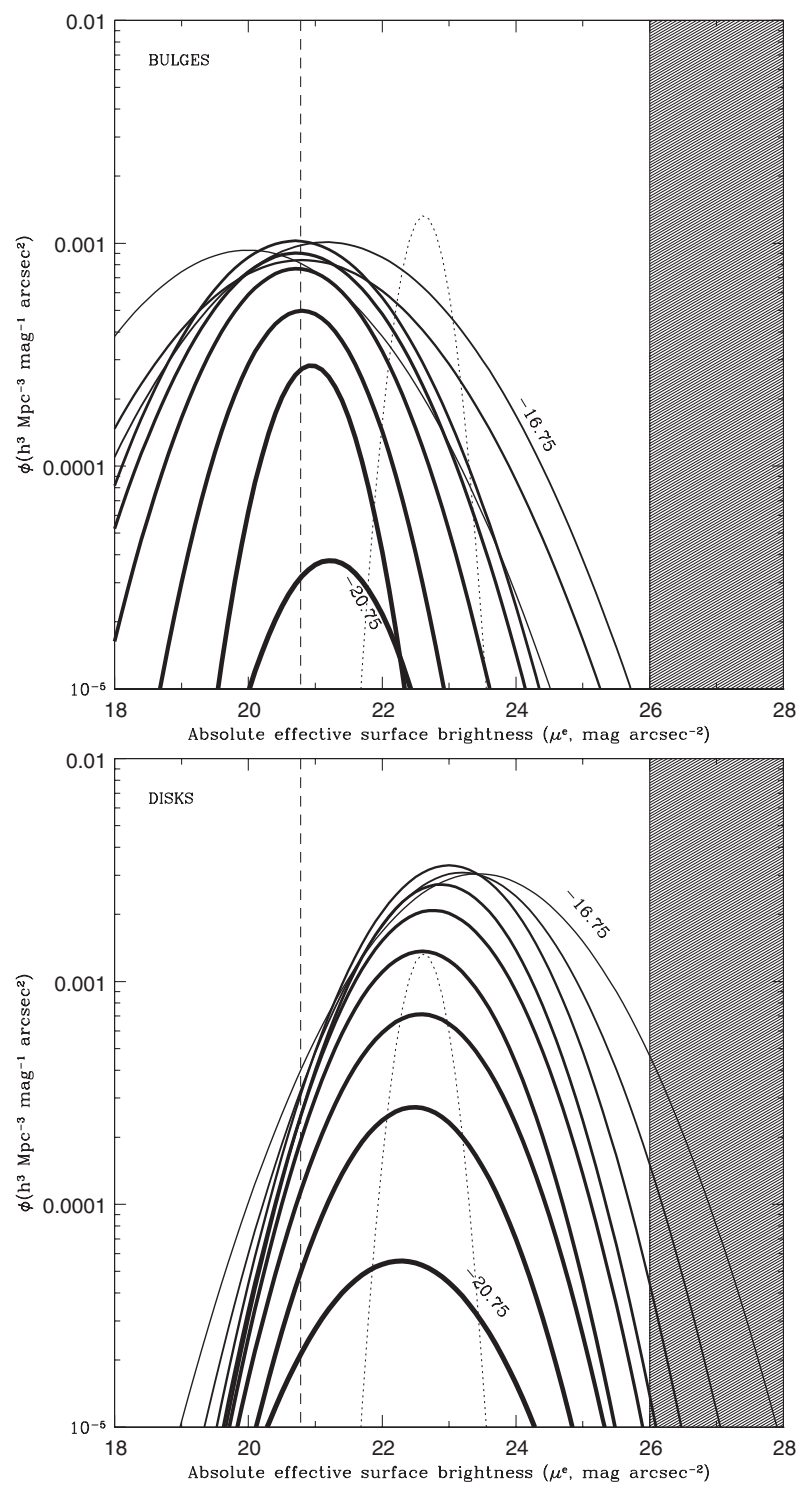

Figure 4 The surface brightness distributions of bulge (top) and disk (bottom) components. The vertical dashed line shows the expected surface brightness for spheriods at $L^{*}\left(M_{B} \approx-19.6 \mathrm{mag}\right)$ and the dotted curve the Freeman distribution for disks systems. The shaded regions show the approximate selection boundaries.

requires robust bulge-disk decompositions of extensive samples over a variety of epochs.

\subsection{The Colour-Luminosity Plane}

The next most obvious key global parameter, after luminosity and surface brightness (size), is colour and, in particular, the rest $-(u-r)$ which straddles the $4000-\AA$ break and is hence a crude indicator of the current starformation rate. Baldry et al. (2004) and Hogg et al. (2004) have recently studied this plane extensively with SDSS data and demonstrate clear bimodality of the colour distribution. Figure 5 shows this trend for the 10000 galaxies of the MGC (using SDSS colours). Figure 5 also shows this trend for the bulge and disks separately. To obtain the bulge colour we use the SDSS PSF magnitudes, and to obtain 


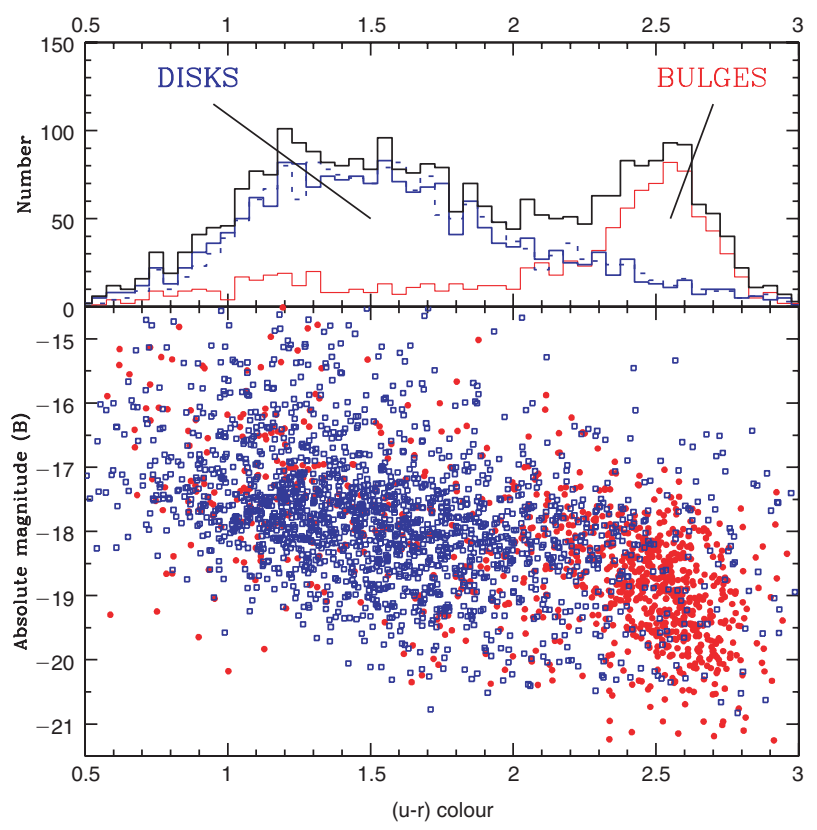

Figure 5 (Top) The bimodal distribution of rest $-(u-r)$ colour (white) subdivided into bulge (red) or disk (blue) components. It is clear that the bimodal distribution is really a bulge-disk dichotomy. (Bottom) The same data shown according to rest $-(u-r)$ colour and absolute $(B)$ magnitude. The sample is not volume-corrected but nevertheless shows that bluer systems are typically of lower luminosity.

the disk colour and we remove the bulge colour component from the global colour to reveal the disk colour ${ }^{5}$. We now see that the bimodal distribution can readily be explained in terms of predominantly red bulges and blue disks. This component segregation implies distinct stellar populations with distinct evolutionary paths. Bulges must contain old stellar population, and disks intermediate or young populations. Again this follows conventional wisdom but highlights yet further the important of bulgedisk decomposition and the need to study the component properties of galaxies rather than the global properties.

\section{Future Prospects}

The two distributions outlined above, both suggest that the well-known bulge and disk components of galaxies follow distinct trends in both the surface brightness (size) and colour distributions. This is of course not particularly new, however what is exciting is our ability to quantify these distributions and trends in detail for large statistical samples, and to extend this kind of structural analysis to higher redshift. In particular the data resolution and signal-to-noise of the ground-based data discussed above is comparable to that available with the Hubble Space Telescope (Driver et al. 1995a, 1995b, 1998b; Driver 1999) and the upcoming James Webb Space Telescope. There is nothing, other than hard diligent work, to prevent us from

5i.e. $\quad(u-r)_{\mathrm{D}}=-2.5 \log \left[10^{\left(-0.4 u_{\mathrm{T}}\right)}-10^{\left(-0.4(u-r)_{\mathrm{B}}\right)}\right]-r_{\mathrm{T}}-2.5 \times$ $\log \left[10^{(1-B / T)}\right]$ where the filter subscript refers to total Petrosian magnitude (T), or disk (D) or bulge (B) PSF magnitude, and $B / T$ refers to the bulge-to-total magnitude, as determined from GIM2D. quantifying the evolution of these distributions across the entire path length of the universe. However three further issues are worth raising:

(1) Which wavelength is optimal for structural studies of galaxies?

(2) How might we push back the boundaries into the dwarf regime?

(3) Can we connect structural measurements to the properties of the dark matter halo?

\subsection{The Near-IR}

Traditionally, almost all nearby galaxy catalogues have been based on flux-limited observations through an optical $B$ or blue bandpass filter $(\sim 400-450 \mathrm{~nm})$, for example the RC3, the 2dFGRS, and the MGC. This has been driven by technological and commercial necessity, with flux detectors typically optimised to the spectral response of the human eye (400-800 nm). However the most physically meaningful bandpass in which to observe a galaxy is in the near-IR (rest $-H$ band or $1.65 \mu \mathrm{m}$ ). This is mostly because the stellar population that dominates the total stellar mass - and therefore best traces a galaxy's gravitational potential - is the long-lived low-mass population which emits in the near-IR (see for example Gavazzi et al. 1996). This is most clearly demonstrated by the obviously smoother appearance of a galaxy in the near-IR than in progressively bluer wavelengths (see upper panel of Figure 6 showing a montage of images for M51 in a variety of filters — the near-IR images (right-most) are significantly smoother. The flux and shape of a galaxy in the near-IR is most dependent on the older relaxed stellar population and therefore a better tracer of the underlying potential. Conversely the flux and shape in the optical is linked to the young stars and therefore dependent on the current and possibly transient star-formation rate. Both optical and near-IR data are important if one wishes to understand galaxy formation and evolution. The near-IR however appears to be the optimal filter for the investigation of the structural properties. The other great advantage is of course the minimisation of the impact of dust obscuration. This is illustrated in the main panel of Figure 6 which shows the location of the $B$ and $H$ band filters superimposed on the night sky spectrum, a galaxy's continuum before and after star burst, and the dust attenuation curve. The impact of star-formation and dust is clearly less in the near-IR. The upcoming near-IR facilities, and in particular UKIRT/WFCAM and VISTA, will have the capabilities to provide exactly the kind of wide, deep, and high-resolution data required for the comprehensive structural analysis of nearby galaxies.

\subsection{The Dwarf Regime}

The space density of dwarf galaxies remains elusive. Figures $1 \& 3$ show that the MGC can only sample with credibility to $M_{B} \approx-16 \mathrm{mag}$, at which point both limiting statistics and the high and low surface brightness selection 

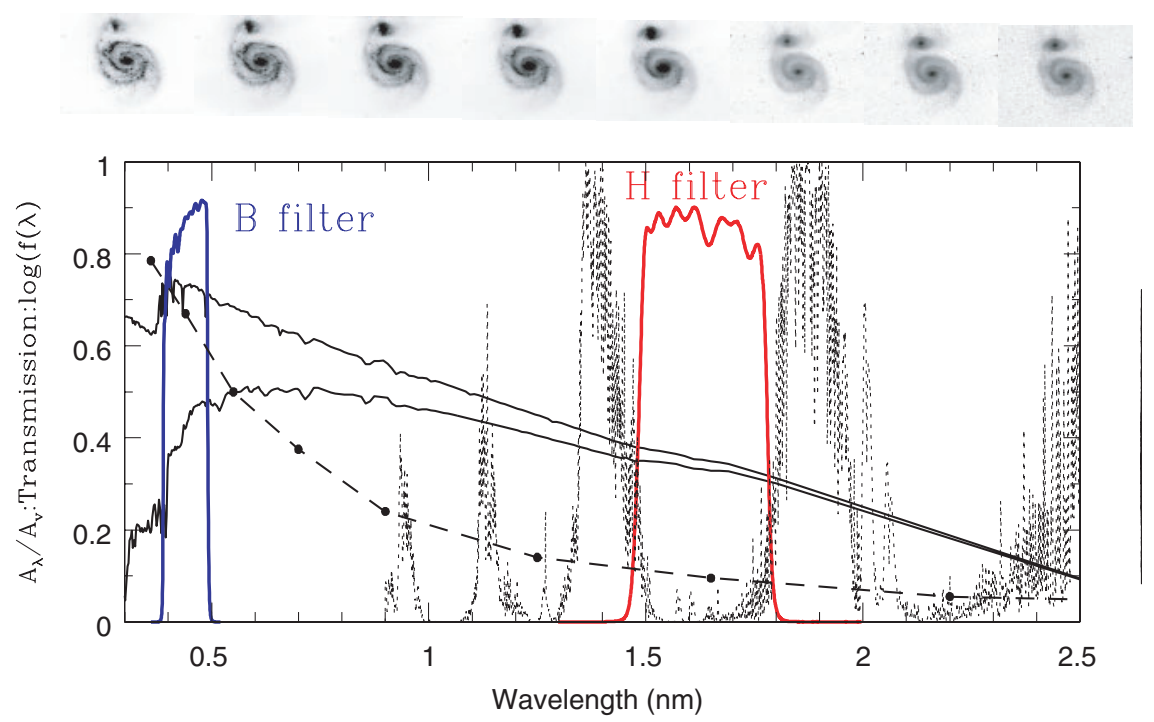

Figure 6 An illustration of the advantages of the near-IR. M51 images in UBVRIJHK respectively are shown along the top. The main panel shows a spectrum of the night sky (dotted line), a spectrum of a galaxy before and after star burst (solid lines), and the location of the $B$ and $H$ filters. The extinction curve is also shown. At shorter wavelengths one has to contend with the vagaries of dust (long dashed line) and star formation. At longer wavelength images are smoother and less affected by dust and star-formation. For detailed structural analysis the longer wavelengths are clearly optimal.

limits bite (see Driver et al. 2004). Figure 7 illustrates this by showing the MGC galaxies on an absolute magnitude versus redshift plot. The data are of course bounded by the $B=20$ mag limit, which highlights the rapidly diminishing volume observed for low luminosity systems. One way to overcome this is to simply conduct ever deeper redshift surveys (as indicated on the Figure). However this has a diminishing return, as the number of galaxies one must observe to find one low luminosity system becomes unreasonable. One possible way forward is to use photometric redshifts to pre-select low- $z$ candidates and then follow only these systems. However the accuracy of photometric redshifts at low $z$ is poor (although improved by near-IR colours, see Bolzonella, Miralles, \& Pelló 2000 for example). To overcome the surface brightness selection limits (both high and low) the source data must be improved to probe to very high resolution $\left(\mathrm{FWHM}<0.5^{\prime \prime}\right.$ ) and very deep isophotes $\left(\mu_{B}>>26 \mathrm{mag} \operatorname{arcsec}^{-2}\right)$ over wide areas $\left(30+\mathrm{deg}^{2}\right)$. No such survey exists but facilities such as SUBARU/SUPrime and Magellan/IMACS just about have the capability to achieve such a survey. The alternative method is to observe the very local galaxy population (i.e. the Local Sphere of Influence, defined as that within $10 \mathrm{Mpc}$ ) and obtain direct distance measurements rather than redshifts.

\subsection{Physics of the LSP?}

The LSP may have the potential to connect key observables (luminosity and size) to the fundamental underlying physical properties of bulge and disk systems (mass and angular momentum). In various studies of the formation of disk systems (e.g. Fall \& Efstathiou 1980; Dalcanton et al. 1997; Mao et al. 1998) the dimensionless spin parameter $\left(\lambda=J\left|E^{1 / 2}\right| G^{-1} M_{\text {halo }}^{-5 / 2}\right.$; Peebles 1969) is directly related

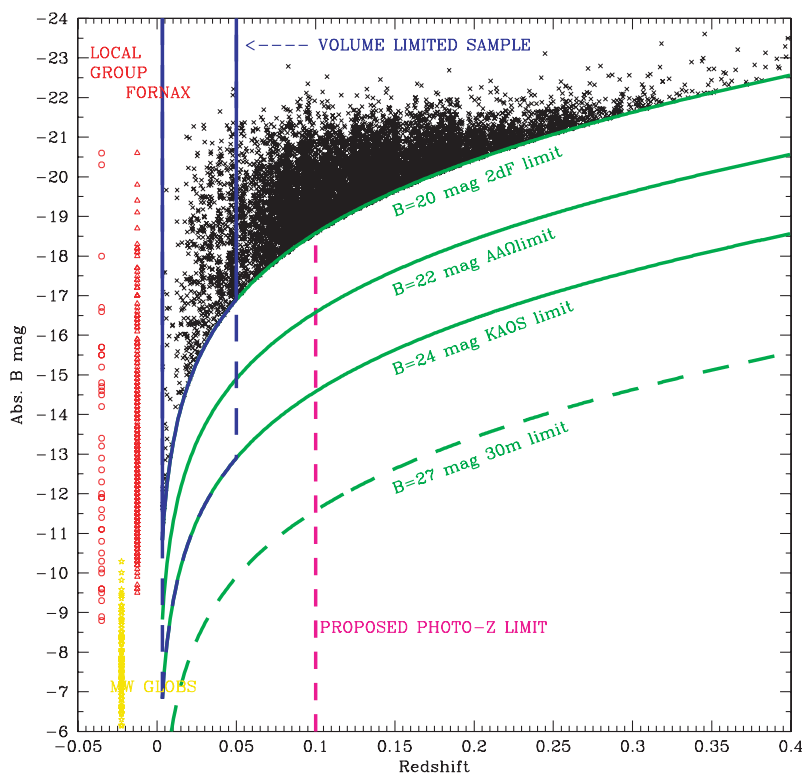

Figure 7 The difficulty of quantifying the faint-end of the luminosity function is highlighted in an $M$ versus $z$ plot such as this one. The data points are from the MGC which is limited at $B=20 \mathrm{mag}$. The yellow box marks its boundary and it barely contains any volume for very low luminosity systems. One possible way forward is to simply push progressively deeper with the upcoming multiplex spectrographs (e.g. AA $\Omega$ on the Anglo Australian Telescope or GMOS/KAOS on Gemini). The main problem with this approach is the sheer numbers of objects. To circumvent this one can envisage implementing a photometric redshift cut first to pre-select candidate low $z$ objects. The left side indicates the distribution of galaxies from the local group, Fornax, and the Milky Way globular cluster distribution as indicated.

to the scale length of the disk. The spin parameter reflects how close the halo is to a rotationally supported system and is a key parameter monitored by the numerical simulations (see Steinmetz \& Bartelmann 1995; Cole \& Lacey 


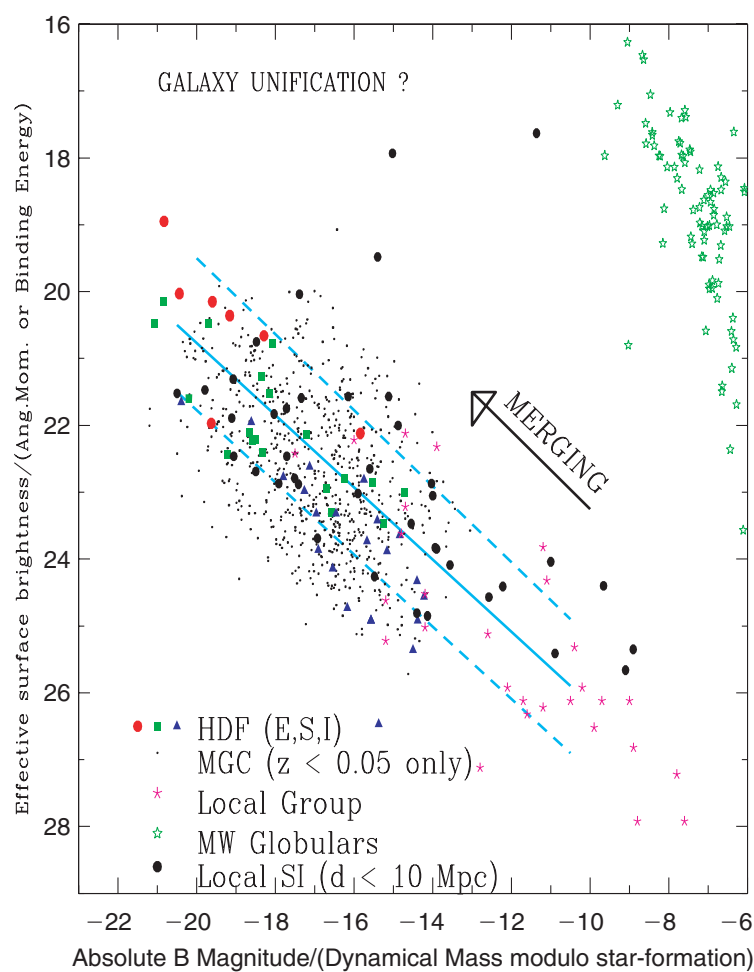

Figure 8 A summary of available LSP data drawn from a variety of sources. The red line marks the credibly mapped area and the cyan line shows the expectation from de Jong \& Lacey (2000). This appears to follow the data remarkably well.

1996; Vitvitska et al. 2002; Maller, Dekel, \& Sommerville 2002 for examples). The pivotal idea (here echoing the toy model of de Jong \& Lacey 2000) starts with the premise that the baryons are coupled to the dark matter halo; because of this the luminosity (generated by the baryons in the form of stars) can be related to the systemic mass and the rotation of the stars/gas can be related to the systemic angular momentum. Given this premise, which is intimated by the Tully-Fisher relation, one can analytically relate $\lambda$ to luminosity and surface brightness (or size) through $\lambda \propto \Sigma_{\text {eff }}^{-1 / 2} L^{-\gamma / 3+1 / 2}$ (from de Jong \& Lacey 2000), where $\Sigma_{\text {eff }}$ is the effective surface brightness, $L$ is the intrinsic luminosity in some filter, and $\gamma$ is the dependence of luminosity on the mass-to-light ratio (equal to 0.69 in $B$ or 1.00 in $H$; Gavazzi et al. 1996). Numerical simulations consistently find that the distribution of the spin parameter is a log-normal distribution, which is globally preserved through hierarchical merging (see for example Vitvitska et al. 2002), to yield $\Sigma_{\text {eff }}=L_{B}^{0.54}$ or $\mu_{\text {eff }}=0.54 M_{B}$. Hence the gradient of any luminosity-surface brightness relation bears upon the relation between mass and light and the dispersion upon the breadth of the spin distribution. Figure 8 shows the $B$-band LSP for a variety of samples as indicated (LG, Mateo 1998; HDF, Driver 1999; MGC, Driver et al. 2004; MW GCs, Harris et al. private communication; Local Sphere of Influence, Jerjen et al. 2000; LSBGs, de Blok, van der Hulst, \& Bothun 1995). The solid lines show the approximate expectation as argued above and show remarkable agreement with the data — in detail the observed size distribution is marginally narrower than simulations predict (see Driver et al. 2004). It is also worth noting that systems which form through merging (i.e. bulges) and through accretion (i.e. disks) are also predicted to show distinct $\lambda$ distributions (see for example Vitvitska et al. 2002; Maller et al. 2002). At the moment far more data and detailed simulations are required, however this connection is clearly promising and could ultimately result in a galaxy equivalent of the Hertzsprung-Russell diagram, allowing a meeting ground between numerical simulations and survey observations.

\section{Summary}

The galaxy luminosity function is not the only fruit, and with the many Legacy datasets becoming available the time is now ripe to move beyond the LF and explore multivariate distributions. Here I've presented two: the luminosity-surface brightness (size) plane and the colourluminosity plane. Both planes show that disks and bulges form distinct but overlapping distributions presumably indicating secular evolution of these components, i.e. two mechanisms and two timescales. This finding argues for the community to move away from global measurements and start to measure the properties of these distinct components independently. I argue that this is best done in the near-IR and should be a key focus of upcoming IR facilities such as VISTA (low- $z$ ) and JWST (high- $z$ ). Perhaps most important of all the LSP appears to provide a direct meeting ground to the numerical simulations. This last point is by far the most important, as it is from the cross-talk between simulations and observations that real insight into the processes of galaxy evolution and formation will come.

\section{Acknowledgments}

I am indebted to my colleagues on the MGC and to the hard work of those that have slaved on the superb 2dFGRS, SDSS, and 2MASS facilities and databases. These data are changing the way we view galaxies in a profound way. Finally I'd like to thank the organisers for a very enjoyable meeting and support from PPARC as a visiting fellow to the University of Bristol where this article was revised.

The Millennium Galaxy Catalogue consists of imaging data from the Isaac Newton Telescope and spectroscopic data from the Anglo-Australian Telescope, the ANU 2.3-m, the ESO New Technology Telescope, the Telescopio Nazionale Galileo, and the Gemini Telescope. The survey has been supported through grants from the Particle Physics and Astronomy Research Council (UK) and the Australian Research Council. The data and data products are publically available from www. eso.org/ jliske/mgc/ or on request from J. Liske or S. P. Driver.

\section{References}

Andreon, S., \& Culliandre, J.-C. 2002, ApJ, 569, 144

Baldry, I. K., Glazebrook, K., Brinkmann, J., Ivezic, Z., Lupton, R. H., Nichol, R. C., \& Szalay, A. S. 2004, ApJ, 600, 681 
Beijersbergen, M., Hoekstra, H., van Dokkum, P. G., \& van der Hulst, T. 2002, MNRAS, 329, 385

Bertin, E., \& Arnouts, S. 1996, A\&AS, 117, 393

Blanton, M. R., et al. 2001, AJ, 121, 2358

Blanton, M. R., et al. 2003, ApJ, 592, 819

Bolzonella, M., Mirralles, J.-M., \& Pelló, R. 2000, A\&A, 363, 476

Bothun, G. D., Impey, C., Mould, J., \& Malin, D. 1987, MNRAS, 94, 23

Chołoniewski, J. 1985, MNRAS, 214, 197

Cole, S., \& Lacey, C. 1996, MNRAS, 281, 716

Colless, M., et al. 2001, MNRAS, 328, 1039

Cross, N. J. G., et al. 2001, MNRAS, 324, 825

Cross, N. J. G., \& Driver, S. P. 2002, MNRAS, 329, 579

Cross, N. J. G., et al. 2004, MNRAS, 349, 576

Croton, D., et al. 2004, MNRAS, in press (astro-ph/0407537)

Dalcanton, J. J. 1998, ApJ, 495, 251

Dalcanton, J. J., Spergel, D., \& Summers, F. J. 1997, ApJ, 482, 659

de Blok, E., van der Hulst, T., \& Bothun, G. D. 1995, MNRAS, 274,235

de Jong, R. S., \& Lacey, C. 2000, ApJ, 545, 781

De Propris, R., et al. 2003, MNRAS, 342, 725

Disney, M. J. 1976, Natur, 263, 573

Driver, S. P., Phillipps, S., Morgan, I., Davies, J. I., \& Disney, M. J. 1994, MNRAS, 268, 393

Driver, S. P., Windhorst, R. A., \& Griffiths, R. E. 1995a, ApJ, 453,48

Driver, S. P., Windhorst, R. A., Ostrander, E. J., Keel, W. C., Griffiths, R. E., \& Ratnatunga, K. U. 1995b, ApJL, 449, 23

Driver, S. P., Couch, W. J., \& Phillipps, S. 1998a, MNRAS, 301, 369

Driver, S. P., Fernandez-Soto, A., Couch, W. J., Odewahn, S. C., Windhorst, R. A., Phillipps, S., Lanzetta, K., \& Yahil, A. 1998b, ApJ, 496, 93

Driver, S. P. 1999, ApJ, 526, 69

Driver, S. P., Odewahn, S. C., Echevarria, L., Cohen, S. H., Windhorst, R. A., Phillipps, S., \& Couch, W. J. 2003, AJ, 126, 2662

Driver, S. P., Liske, J., Cross, N. J. G., De Propris, R., \& Allen, P. D. 2004, MNRAS, submitted

Driver, S. P., \& De Propris, R. 2003, Ap\&SS, 285, 175
Fall, M., \& Efstathiou, G. 1980, MNRAS, 193, 189

Freeman, K. C. 1970, AJ, 160, 811

Gavazzi, G., Pierrini, D., \& Boselli, A. 1996, 312, 397

Hogg, D. W., et al. 2004, ApJL, 601, 29

Impey, C., \& Bothun, G. D. 1988, ApJ, 330, 634

Impey, C., \& Bothun, G. D. 1997, ARA\&A, 35, 267

Jarrett, T. H., Chester, T., Cutri, R., Schneider, S. E., \& Huchra, J. P. 2003, AJ, 125, 525

Jerjen, H., Binggeli, B., \& Freeman, K. C. 2000, ApJ, 119, 593

Kormendy, J. 1977, ApJ, 218, 333

Liske, J., Lemon, D., Driver, S. P., Cross, N. J. G., \& Couch, W. J. 2003, MNRAS, 344, 307

Maller, A. H., Dekel, A., \& Sommerville, R. 2002, MNRAS, 329,423

Mateo, M. 1998, ARA, 36, 435

Mao, S., Mo, H. J., \& White, S. D. M. 1998, MNRAS, 297, 71

Mobasher, B., et al. 2004, MNRAS, 587, 605

Norberg, P., et al. 2002, MNRAS, 336, 907

Peebles, J. 1969, ApJ, 155, 393

Press, W., \& Schechter, P. J. 1974, ApJ, 187, 425

Pracey, M. B., De Propis, R., Driver, S. P., Couch, W. J., \& Nulsen, P. E. J. 2004, MNRAS, 352, 1135

Schechter, P. 1976, ApJ, 203, 297

Shen, S., Mo, H. J., White, S. D. M., Blanton, M. R., Kauffmann, G., Voges, W., Brinkmann, J., \& Csabai, I. 2003, MNRAS, 343, 978

Simard, L., et al. 2002, ApJS, 142, 1

Smith, R. M., Driver, S. P., Phillipps, S., \& Couch, W. J. 1997, MNRAS, 287, 415

Sodré, L., Jr., \& Lahav, O. 1993, MNRAS, 260, 285

Sprayberry, D., Impey, C., Irwin, M. J., \& Bothun, G. D. 1997, ApJ, 482,104

Steinmetz, M., \& Bartelmann, M. 1995, MNRAS, 272, 570

Stoughton, C., et al. 2002, AJ, 123, 485

Trentham, N. 1998, MNRAS, 293, 71

Trentham, N., \& Hodgkin, S. 2002, MNRAS, 333, 423

Vitvitska, M., Klypin, A. A., Kravtsov, A. V., Wechsler, R. H., Primack, J. R., \& Bullock, J. S. 2002, ApJ, 581, 799 